\title{
Synergetic effects of hBMSCs and hPCs in osteogenic differentiation and their capacity in the repair of critical-sized femoral condyle defects
}

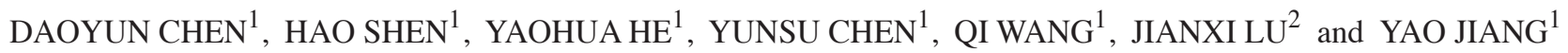 \\ ${ }^{1}$ Department of Orthopaedic Surgery, Shanghai Sixth People's Hospital, Shanghai Jiaotong University, \\ Shanghai 200233; ${ }^{2}$ Department of Orthopedic Surgery, Shanghai Ninth People's Hospital, \\ Shanghai Jiaotong University, Shanghai 200011, P.R. China
}

Received September 15, 2013; Accepted April 28, 2014

DOI: $10.3892 / \mathrm{mmr} .2014 .2883$

\begin{abstract}
Tissue-engineered bone grafts require an osteoblastic cellular source to be utilized in bone transplantation therapy. Human bone marrow stem cells (hBMSCs) and periosteal-derived stem cells (hPCs) are the commonly used cellular sources for bone tissue engineering and are essential in fracture healing. In the present study, hBMSCs and hPCs were co-cultured from the same donors, as the cellular source. In monolayer cultivation, co-culturing hBMSCs and hPCs demonstrated more robust mineralized nodule formation and stronger alkaline phosphatase (ALP) positive staining than hBMSCs or hPCs. Three-dimensional (3-D) culturing on porous $\beta$-tricalcium phosphate (TCP) scaffolds and co-culturing of hBMSCs and hPCs significantly promoted the osteogenic specific mRNA expression of COL1 $\alpha 1$, BMP-2, osteopontin (OPN) and osteocalcin (OC). For in vivo bone formation and neovascularization assessment, the cellular- $\beta$-TCP scaffolds were transplanted into critical-sized femoral condyle defects in rabbits. The results confirmed that co-culturing hBMSCs and hPCs accelerated bone regeneration and enhanced mature bone formation, but also facilitated central vascularization in scaffold pores. Based on these data, we recommend co-culturing hBMSCs and hPCs as a promising cellular source for bone tissue engineering applications.
\end{abstract}

Correspondence to: Professor Yao Jiang or Dr Yaohua He, Department of Orthopaedic Surgery, Shanghai Sixth People's Hospital, Shanghai Jiaotong University, 600 Yishan Road, Shanghai 200233, P.R. China

E-mail: jiangyaosjtu@sina.com

E-mail: heyaohua@sjtu.edu.cn

Key words: co-culture, human bone marrow mesenchymal stem cells, human periosteal-derived stem cells, critical-sized femoral condyle defects, bone tissue engineering, osteogenesis, neovascularization

\section{Introduction}

Critical-sized bone defects often demand the transplantation of bone tissue or substitutes, to restore bone integrity. The gold standard method for orthopedic surgical procedures is the use of autologous bone grafts to stimulate bone growth and implant fixation. However, limited quantities of bone are available for autografting and the harvest procedure involves potential donor site morbidity (1). While allografted bone has been widely used, it is limited by the associated risks, including immunogenicity and transmission of infectious diseases $(2,3)$. Bone tissue engineering is a promising approach to overcome these limitations.

One strategy utilized to repair bone defects by bone tissue engineering, involves the combination of osteogenic cells with the appropriate porous absorbable scaffolds. In this cell-based therapy, mesenchymal stem cells (MSCs) are regarded as an excellent cellular source for bone tissue engineering because of their self-replication and osteogenic differentiation capacities $(4,5)$. A variety of adult MSCs have been isolated from a diverse range of tissue types and ontogenies, including bone marrow, periosteum, synovium, umbilical cord blood, amniotic fluid, liver and adipose tissue (6-12). Among these, bone marrow and periosteum are the most commonly used cellular source for bone regeneration therapy. However, each source has its disadvantages, including the fact that MSCs isolated from bone marrow have limited proliferation capacity and high cellular senescence, and their osteogenic potential decreases with age $(13,14)$. Furthermore, periosteal-derived stem cells isolated from different donor sites and species have been reported to demonstrate wide viability in osteogenic potential $(15,16)$. Thus, the correct selection of MSCs as a cell source is of high importance in constructing engineered bone tissue.

It is well established that fracture healing requires the mobilization of MSCs, to allow deposition of cartilage and bone at the injury site. These cells are considered to be recruited locally and concurrently from the periosteum and bone marrow during bone repair. Although the periosteum and bone marrow generate osteoblasts, these cell types have demonstrated distinct cellular responses in the process of 
bone healing and it has been confirmed that the periosteum is critical in new bone tissue mineralizaion $(17,18)$. Furthermore, it has also been identified that injured periosteum and bone marrow heal in a different manner. Periosteum injuries heal by endochondral ossification, whereas bone marrow injuries heal by intramembranous ossification $(17,19)$.

However, whether MSCs isolated from the bone marrow and periosteum have synergistic effects on osteogenic potential remains unclear. In the present study, hBMSCs with hPCs from the same donors were co-cultured with the aim of determining whether this strategy would accelerate the osteogenic potential of MSCs. For in vitro evaluation, alizarin red $\mathrm{S}$ and ALP staining were used for monolayer cultivation, and osteogenic-specific mRNA expression was tested in threedimensional (3-D) cultivation. For in vivo assessment, the MSCs from each group were seeded onto porous $\beta$-tricalcium phosphate (TCP) scaffolds and transplanted to critical-sized femoral condylar defects in rabbits, and the bone formation volume, mature bone percentage and blood vessel ingrowth were subsequently determined.

\section{Materials and methods}

Samples, animals and ethics. Human bone marrow and periosteum samples were obtained from patients undergoing lower limb amputation surgery because of severe limb trauma. Samples were obtained from 8 healthy donors (six males and two females; range, 22-30 years of age) in accordance with the local ethics committee and after obtaining informed consent. The bone marrow was harvested from the inferior segment of the tibia. During the same surgical procedure, the periosteum was harvested from the distal part of the tibia.

New Zealand rabbits ( $\mathrm{n}=36$; weighing, 2.5 $\pm 3.2 \mathrm{~kg}$ ) were provided by the Laboratory Animal Centre of the Sixth People's Hospital of Shanghai Jiaotong University (Shanghai, China). All procedures were approved by the Animal Care and Use Committee of Shanghai Sixth People's Hospital, Shanghai Jiaotong University (Shanghai, China).

Isolation of hBMSCs. The isolation of hBMSCs was performed as previously described (20). Briefly, a single-cell suspension was passed through an $80 \mu \mathrm{m}$ cell strainer (BD Biosciences, San Diego, CA, USA). The cells were then plated in $25 \mathrm{~cm}^{2}$ culture flasks and cultured in a complete medium (CM) consisting of Dulbecco's modified Eagle's medium (Gibco, Gaithersburg, MD, USA), supplemented with $10 \%$ fetal bovine serum (Gibco), $100 \mathrm{U} / \mathrm{ml}$ penicillin and $100 \mu \mathrm{g} / \mathrm{ml}$ streptomycin at $37^{\circ} \mathrm{C}$ in $95 \%$ humidified air and $5 \% \mathrm{CO}_{2}$. Non-adherent cells were removed by changing the medium twice a week. When the hBMSCs reached 80-90\% confluence, the adherent cells were detached with $0.25 \%$ trypsin/EDTA (Gibco) and subcultured at a density of $1 \times 10^{4} / \mathrm{cm}^{2}$ in $25 \mathrm{~cm}^{2}$ culture flasks.

Isolation of hPCs. The culture of hPCs was performed as described previously (21). Following rinsing the periosteum thoroughly with phosphate-buffered saline (PBS) containing $100 \mathrm{U} / \mathrm{ml}$ penicillin and $100 \mu \mathrm{g} / \mathrm{ml}$ streptomycin, the biopsy specimens were minced into small pieces and digested in $0.2 \%$ type II collagenase (Sigma, St. Louis, MO, USA) for $4 \mathrm{~h}$ at $37^{\circ} \mathrm{C}$. The isolated cells were centrifuged and resuspended in the $\mathrm{CM}$ at $37^{\circ} \mathrm{C}$ in $95 \%$ humidified air and $5 \% \mathrm{CO}_{2}$. The hPCs were subcultured as described above for the hBMSCs. The hBMSCs and hPCs at passages 3 were used in the experiments.

Co-culture design of hBMSCs and hPCs. The hBMSCs and hPCs from the same donors were used for co-culture experiments with three different ratios. The ratio of hBMSCs to hPCs in group 1 was 1:1; in group 2 was 1:2 and in group 3, was $2: 1$. For the monolayer culture, three types of co-cultured MSCs, hBMSCs and hPCs were seeded at a density of 5,000 cells $/ \mathrm{cm}^{2}$ into 6-well dishes with CM. Following $24 \mathrm{~h}$ in culture, the medium was replaced by an osteogenic medium consisting of CM supplemented with $10 \mathrm{nM}$ dexamethasone (Sigma), $0.05 \mathrm{mM}$ L-ascorbic acid 2-phosphate (Sigma) and $10 \mathrm{mM} \beta$-glycerophosphate (Sigma). The cells were cultured in osteogenic medium for three weeks and the medium was changed twice a week.

\section{Osteogenic differentiation in monolayer cultures}

ALP staining. Following rinsing of the monolayer cells with PBS, they were fixed in an ice-cold, $90 \%$ ethanol solution for $10 \mathrm{~min}$ and washed in PBS for $5 \mathrm{~min}$. Then, the cells were stained with fast 5-bromo-4-chloro-3-indolyl phosphate and nitroblue tetrazolium (BCIP/NBT) ALP substrate (Beyotime Biotechnology, Jiangsu, China) for $30 \mathrm{~min}$ at room temperature. The reaction was terminated by removing the substrate solution and washing with distilled water. The results were expressed as the percentage of positive staining area per field of view (magnification, x100).

Alizarin red S staining. For the mineralized nodule formation assay, the mineralized matrix was analyzed using alizarin red $\mathrm{S}$ staining. The cell cultures were rinsed with PBS and fixed in ice-cold, $90 \%$ ethanol solution for $10 \mathrm{~min}$. The cells were washed with distilled water, treated with a $2 \%$ alizarin red $\mathrm{S}$ solution (Amresco, Solon, OH, USA) for $5 \mathrm{~min}$ and washed with distilled water to remove the remaining staining. The results were expressed as the percentage of positive staining area in per field of view (magnification, x100).

Scaffold preparation and MSCs in 3-D cultures. The $\beta$-TCP scaffolds (Bio-lu Biomaterials, Shanghai, China) were molded into a circular cylinder (6 $\mathrm{mm}$ diameter and $10 \mathrm{~mm}$ length) with a porosity of $70 \%$ and pore diameter of $450 \pm 50 \mu \mathrm{m}$. The scaffolds were sterilized by $60 \mathrm{Co}$ irradiation prior to use. The MSCs were suspended in a fibrin gel (Sigma) and statically loaded into porous $\beta$-TCP scaffolds $\left(1.2 \times 10^{6}\right.$ cells/scaffold $)$, as described previously (21). The cellular scaffolds were cultured in 6-well plates with CM overnight prior to being transferred to the osteogenic medium, which was changed twice a week. For the in vitro osteogenic gene expression assay, the cellular scaffolds were harvested on days 3, 7, 14 and 21 for quantitative polymerase chain reaction (qPCR) analysis. For in vivo evaluation, the cellular scaffolds were pre-differentiated in an osteogenic differentiation medium for 21 days prior to implantation.

$q P C R$. The total cellular RNA on the scaffolds was extracted using TRIzol reagent (Invitrogen Life Technologies, Carlsbad, 
CA, USA) according to the manufacturer's instructions. The concentration of RNA was determined from the optical absorbance at $260 \mathrm{~nm}$ of the extract. Complementary DNA (cDNA) was synthesized using the PrimeScript First Strand cDNA Synthesis kit (TaKaRa Biotechnology, Inc., Dalian, China). Reactions were performed and monitored in a PTC 200 Thermal Cycler PCR machine (Bio-Rad, Waltham, MA, USA). qPCR was performed using a quantitative real-time amplification system (Light Cycler 480; Roche Diagnostics (Schweiz) AG, Risch, Switzerland). SybrGreen Premix Ex TaqII (TaKaRa Biotechnology, Inc.) was used in each reaction. Reactions were performed with 40 cycles $\left(95^{\circ} \mathrm{C}\right.$ for $5 \mathrm{sec}, 55^{\circ} \mathrm{C}$ for $30 \mathrm{sec}$ and $72^{\circ} \mathrm{C}$ for $30 \mathrm{sec}$ ). The primers used for qPCR were as follows: BMP-2, 5'-TGGAAGTGGCCCATTTAGAG-3', 5'-TGACGCTTTTCTCGTTTGTG-3'; Collagen typeIalpha1 (COL $1 \alpha 1), \quad 5$ '-CCT GCGTGTACCCCACTCA-3', 5'-ACCAGACATGCCTCTTGTCCTT-3'; Osteopontin (OPN), 5'-AC ACATATGATGGCCGAGGTGA-3', 5' - TGTGAGGTGATGTCCTCGTCTGTA G-3'; Osteocalcin (OC), 5'-CAAAGGTGCAGCCTTTGTGTC-3', 5'-TCACAGTCCGGATTGAGCTCA-3'; GAPDH, $5^{\prime}$ - G C A C C G T C A A G G C T G A G A C - 3' and 5'-ATGGTGGTGAAGACGCCAGT-3'. Results were normalized against the housekeeping gene GAPDH and relative gene expression was analyzed with the $2-\Delta \Delta \mathrm{Ct}$ method. The human osteoblasts were obtained as the control cell types. Each measurement was assessed in triplicate.

Surgical procedure. The animal model was adapted from Giavaresi et al, as described previously (22). Briefly, following induction of general anesthesia, transversal, critical-sized bone defects were created (6 $\mathrm{mm}$ diameter, $10 \mathrm{~mm}$ length) in the femoral distal epiphysis of the posterior limbs by a standardized surgical procedure. A $2 \mathrm{~cm}$ skin incision was established on the lateral aspect of the distal femoral condyle. Bilateral confined cancellous defects were stepwise drilled in both limbs with a $3.2 \mathrm{~mm}$ drill and these defects were subsequently expanded with a $6.0 \mathrm{~mm}$ drill. The depth of the defects was $10 \pm 0.5 \mathrm{~mm}$ as measured by a digital caliper. The soft tissues were sutured with Dexon 3-0 and the skin was closed with silk 3-0. Analgesics (carprofen, $4 \mathrm{mg} / \mathrm{kg}$ ) were prescribed in the immediate post-operative period. Antibiotic therapy (cefazolin, $25 \mathrm{mg} / \mathrm{kg}$ ) was administered pre-operatively and for five days following surgery. Six experimental conditions were used: (i) hBMSCs, (ii) hPCs, (iii) co-culturing hBMSCs and hPCs with 1:1 ratios, (iv) co-culturing hBMSCs and hPCs with 1:2 ratios, (v) co-culturing hBMSCs and hPCs with 2:1 ratios and (vi) blank $\beta$-TCP scaffolds. A sample size of $n=6$ defect sites per group per time point were examined. Following four and 12 weeks, respectively, the animals were euthanized and the implants were retrieved for histological analysis.

Quantification of newly formed bone. To evaluate the total new bone formation and the mature bone volume, the harvested femoral condyles were fixed in $4 \%$ formaldehyde, decalcified and embedded in paraffin wax. Three middle sections $(5 \mu \mathrm{m}$ thickness) of each implant were stained with hematoxylin and eosin (H\&E) for total new bone tissue area and Van Geison's for mature bone volume. The results were observed under a light microscope (magnification, x100) and at least ten images were randomly obtained in one section. Using image analytical software Image-Pro Plus (Media Cybernetics, Bethesda, MD, USA), the total new bone volume was expressed as a percentage of newly formed bone area in the total cross sectional area and the mature bone volume was calculated as a percentage of mature bone area of the total new bone tissue area.

Neovasculogenesis analysis. To determine the extent of blood vessel ingrowth, the middle sections of the implants were immunostained for vWF (Biosynthesis Biotechnology, Beijing, China), a protein present in large quantities in subendothelial matrices, including blood vessel basement membranes (20). Circular vWF staining was obtained to indicate a blood vessel subendothelium. Blood vessels were counted manually with Adobe Photoshop 8.0 software (Adobe Systems, Mountain View, CA, USA) and were assessed by the mean blood vessel numbers per pore and percentage of blood vessels in the pore center region.

Statistical analyses. Parametric data are represented as the mean $\pm \mathrm{SD}$, analyzed using one-way ANOVA. A value of $\mathrm{P}<0.05$ was considered to indicate a statistically significant difference.

\section{Results}

Osteogenic differentiation capacity in monolayer cultures. As demonstrated by alizarin red $\mathrm{S}$ staining, when exposed to osteogenic medium, the mineralized nodule was generated abundantly in the co-culture condition, compared with the hBMSC and hPC groups (Fig. 1A and C). Furthermore, co-cultured MSCs formed alizarin red $\mathrm{S}$ positive mineralization nodules earlier than hBMSCs and hPCs, which was first observed at day 8 in all three co-cultured MSCs and at day 10 in both hBMSCs and hPCs. The more robust osteogenic differentiation was also confirmed by ALP staining. ALP-positive staining in the early stage was confirmed in all five types of MSCs, although the staining was slightly decreased on day 21 in hBMSCs and hPCs. Co-culturing hBMSCs and hPCs did however, demonstrate a relatively strong positive staining in the early stage (day 3) and in the late stage (day 21), compared with hBMSCs and hPCs (Fig. 1B and D).

Osteogenic gene expression in 3-D cultures. During culturing in the osteogenic medium, hBMSCs, hPCs and co-cultured MSCs of the three different ratios, were assessed for mRNA expression of gene encoding. qPCR demonstrated that co-culturing MSCs in the 3-D model upgraded mRNA expression of COL1 $\alpha 1$, BMP2, OPN and OC at different time points (Fig. 2). High expression of COL1 $\alpha 1$ mRNA was recorded in the early differentiation stage, but a decrease was observed in the late stage. Co-culturing hBMSCs and hPCs significantly increased COL1 1 1 expression at days 3 and 7, respectively (Fig. 2B). OPN was expressed in the early differentiation stage and this effect progressively increased with time. The co-cultured MSCs upgraded the OPN mRNA transcript on days 3 and 7 (Fig. 2B). BMP-2 and OC mRNA transcript was highly expressed at day 14 , which steadily increased at 21 days. Co-culturing significantly enhanced the expression of BMP-2 and OC in the late stage, which were confirmed in all three co-culturing MSCs (Fig. 2C and D). 
A

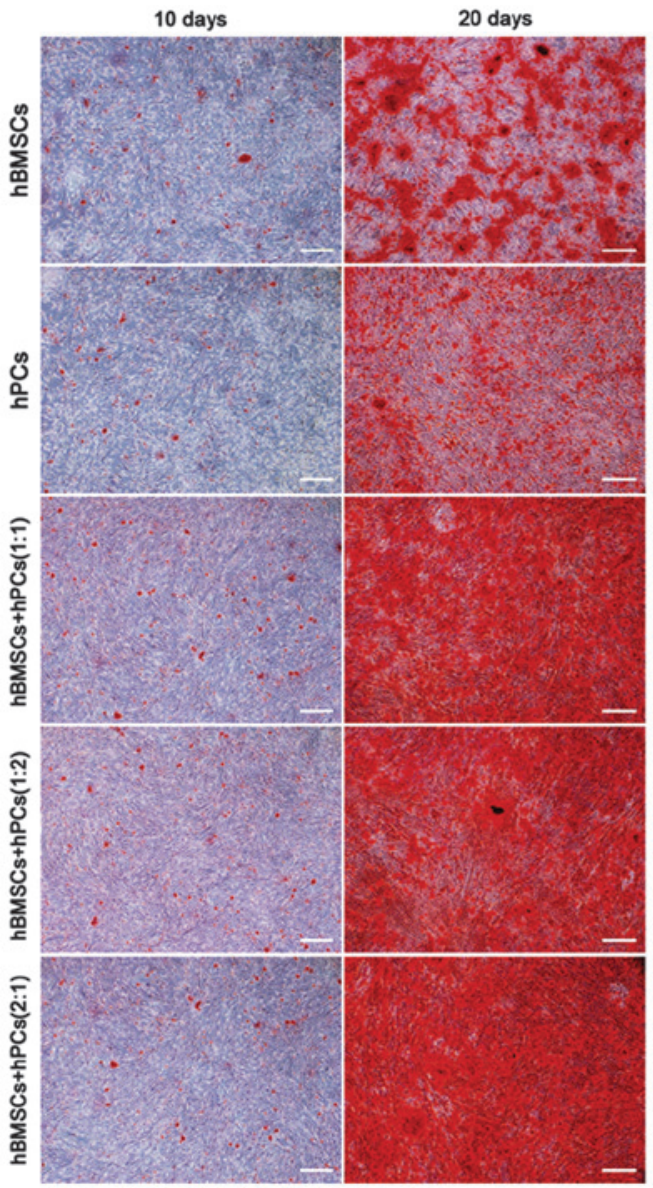

B

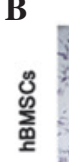

3 days

7 days

14 days

21 days

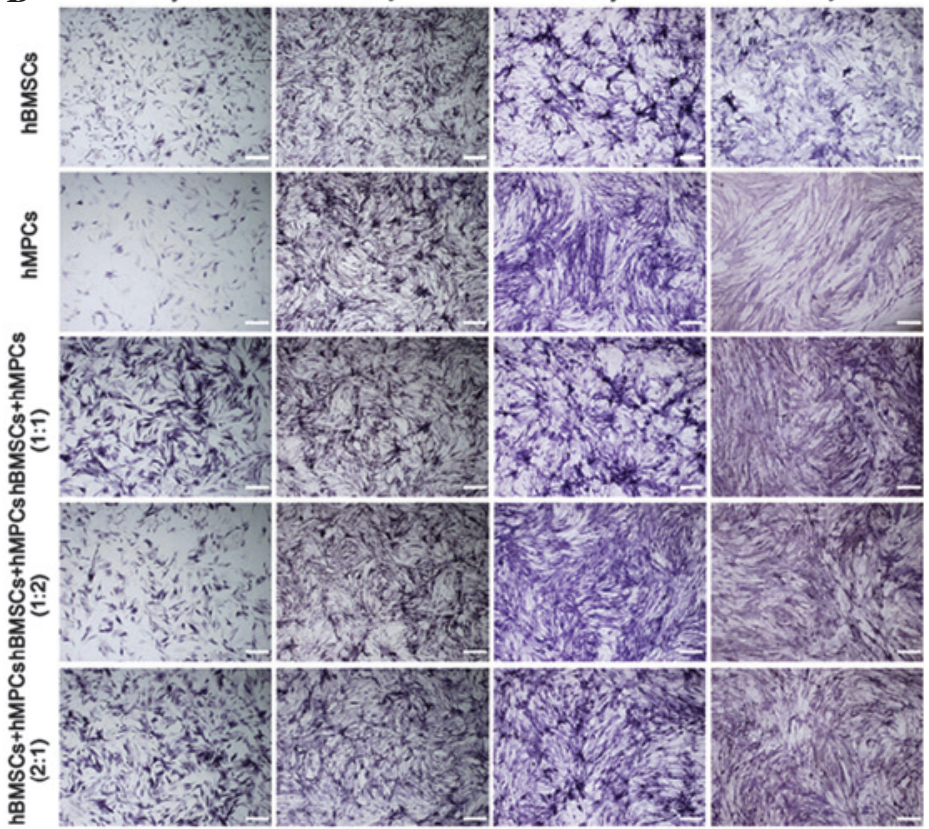

$\mathbf{C}$

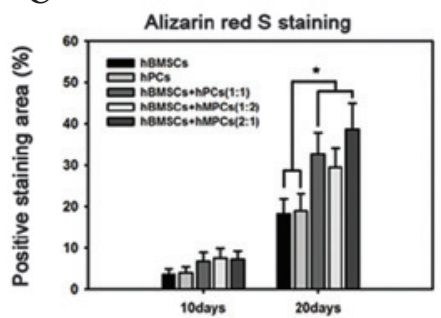

D

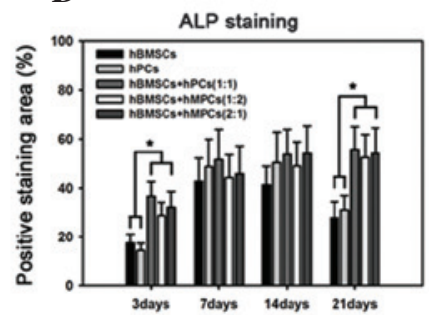

Figure 1. Osteogenic differentiation capacity in monolayer culture. (A) Alizarin red S staining for mineralized nodule formation at culture time of 10 days and 20 days. (B) ALP staining on culture time of 3, 7, 14 and 21 days (bar, $200 \mu \mathrm{m}$ ). (C) The results of alizarin red S staining were expressed as the percentage of positive staining area per field of view (magnification, x100). (D) The results of ALP staining were expressed as the percentage of positive staining area per field of view (magnification, x100). hBMSCs, human bone marrow stem cells; hPCs, human periosteal-derived stem cells.
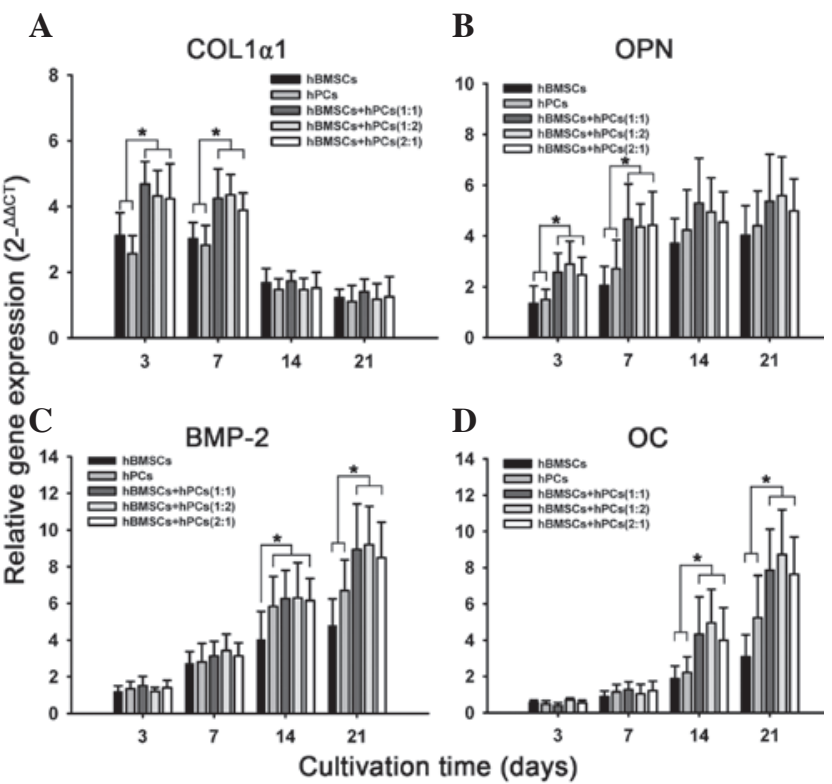

Figure 2. Osteogenic-specific mRNA expression of (A) COL1 $\alpha 1$, (B) OPN (C) BMP-2 and (D) OC in 3D cultivation ( ${ }^{*} \mathrm{P}<0.05$ vs. hBMSCs or/and $\left.\mathrm{hPCs}\right)$. OPN, osteopontin; OC, osteocalcin; COL1 $\alpha 1$, collagen typeIalpha1; 3D, 3-dimensional; hBMSCs, human bone marrow stem cells; hPCs, human periosteal-derived stem cells.
Histological analysis. The cellular-scaffold constructs in the femoral condyle were retrieved for histological evaluation following 4 and 12 weeks' implantation. The total new bone formation in the 3-D $\beta$-TCP scaffolds was indicated by H\&E staining (Fig. 3A). Mature bone volume in the six groups of implants was revealed by Van Geison's staining (Fig. 3B). As summarized in Table 1, the $\beta$-TCP scaffolds seeded with all three different ratios of co-culturing MSCs, significantly increased new bone formation, compared with the scaffolds loaded with hBMSCs and hPCs. The most evident difference in newly formed bone tissue was observed at four weeks. This indicated that this synergetic effect in bone formation was initiated from the early stages of osteogenic differentiation. Furthermore, mature bone volume analysis confirmed that, compared with the hBMSCs and hPCs, co-culturing MSCs exhibited a significantly higher percentage of mature bone formation in the critical-sized femoral condyle defects.

Neovasculogenesis analysis. The effects of blood vessel ingrowth in the 3-D $\beta$-TCP scaffolds were determined by immunostaining tissue sections for vWF (Fig. 4), a component of blood vessel extracellular matrix (ECM). There was a marked increase in immunostained vessels in the TCP 
Table I. Bone formation area and mature bone percentage of engineering bone in critical-sized femoral condyle defects.

\begin{tabular}{|c|c|c|c|c|}
\hline \multirow[b]{2}{*}{ Group } & \multicolumn{2}{|c|}{ Bone formation area $(\%)$} & \multicolumn{2}{|c|}{ Mature bone volume (\%) } \\
\hline & 4 weeks & 12 weeks & 4 weeks & 12 weeks \\
\hline hBMSCs & $25.09 \pm 3.56$ & $36.82 \pm 5.38$ & $36.48 \pm 4.89$ & $48.47 \pm 4.72$ \\
\hline hPCs & $26.58 \pm 4.89$ & $38.73 \pm 7.14$ & $39.31 \pm 5.12$ & $63.43 \pm 4.77^{\mathrm{a}, \mathrm{b}}$ \\
\hline hBMSCs+hPCs $(1: 1)$ & $40.30 \pm 6.83^{\mathrm{a}, \mathrm{b}}$ & $46.83 \pm 7.36^{\mathrm{a}, \mathrm{b}}$ & $48.46 \pm 4.33^{\mathrm{a}, \mathrm{b}}$ & $76.42 \pm 5.82^{\mathrm{a}, \mathrm{b}}$ \\
\hline hBMSCs+hPCs $(1: 2)$ & $38.53 \pm 7.15^{\mathrm{a}, \mathrm{b}}$ & $47.39 \pm 6.88^{\mathrm{a}, \mathrm{b}}$ & $51.54 \pm 3.79^{\mathrm{a}, \mathrm{b}}$ & $76.85 \pm 6.43^{\mathrm{a}, \mathrm{b}}$ \\
\hline hBMSCs+hPCs $(2: 1)$ & $37.58 \pm 7.87^{\mathrm{a}, \mathrm{b}}$ & $45.65 \pm 8.21^{\mathrm{a}, \mathrm{b}}$ & $48.48 \pm 5.17^{\mathrm{a}, \mathrm{b}}$ & $74.15 \pm 5.38^{\mathrm{a}, \mathrm{b}}$ \\
\hline blank $\beta$-TCP scaffold & $15.22 \pm 3.15^{\mathrm{a}, \mathrm{b}}$ & $26.32 \pm 5.41^{\mathrm{a}, \mathrm{b}}$ & $33.70 \pm 4.82$ & $44.18 \pm 4.79^{b}$ \\
\hline
\end{tabular}

${ }^{\mathrm{a}} \mathrm{P}<0.05$ vs hBMSCs; ${ }^{\mathrm{P}}<0.05$ vs hPCs. hBMSCs, human bone marrow stem cells; hPCs, human periosteal-derived stem cells; $\beta$-TCP, $\beta$-tricalcium phosphate.

A

Hematoxylin and eosin staining
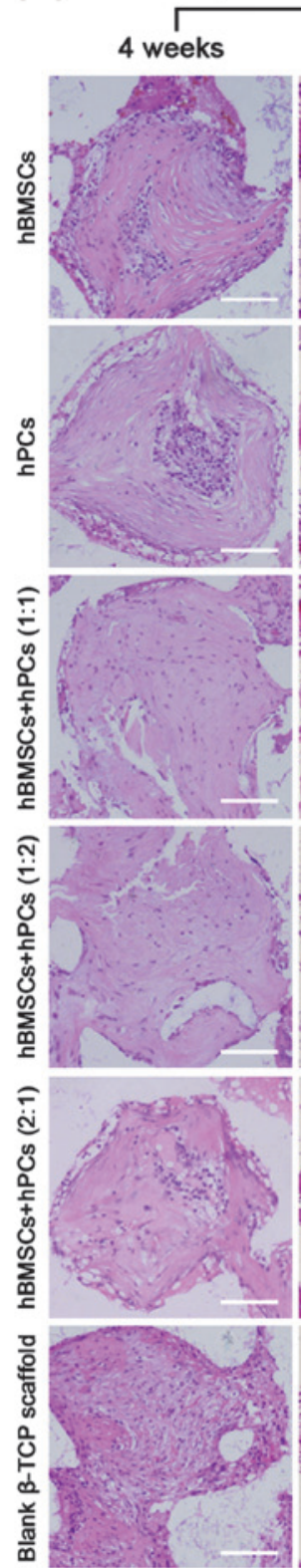
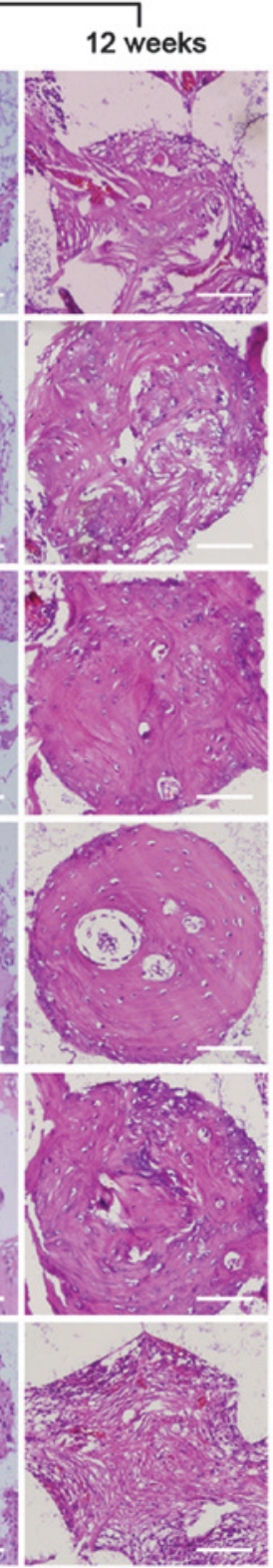

B
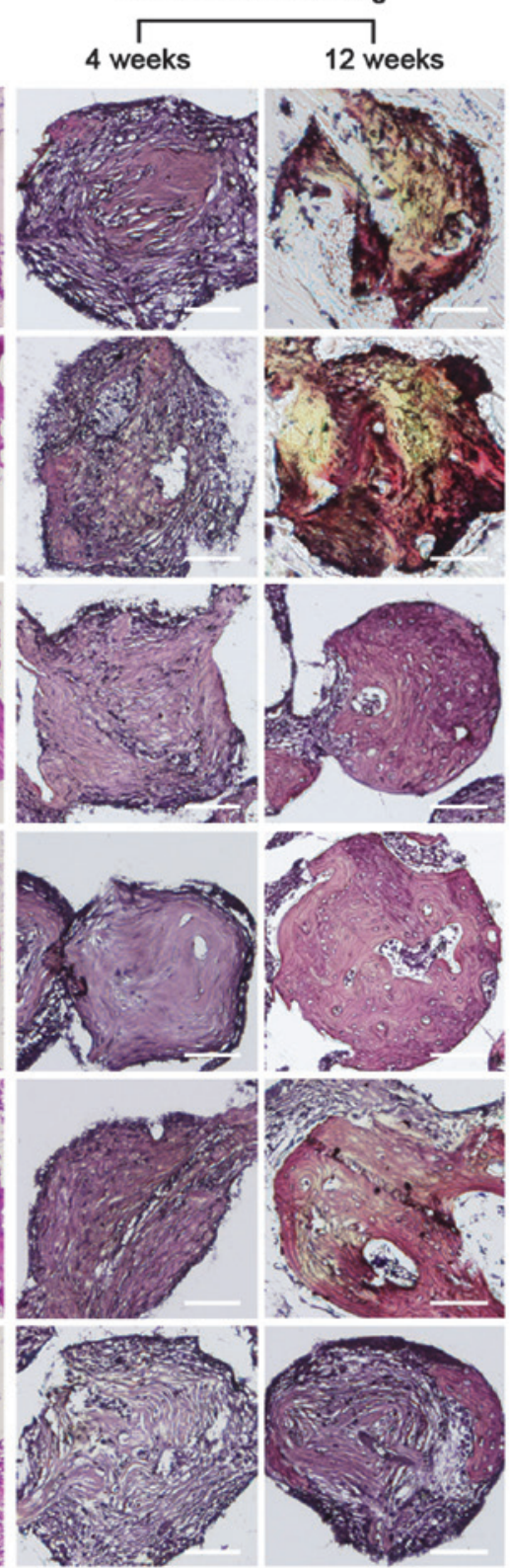

Figure 3. Histological sections of femoral condyle implants harvested following 4 and 12 weeks' implantation. (A) Total new bone formation was determined by H\&E staining. (B) Mature bone volume was determined by Van Geison's staining (bar, $100 \mu \mathrm{m}$ ). hBMSCs, human bone marrow stem cells; hPCs, human periosteal-derived stem cells; H\&E, hematoxylin and eosin. 
Table II. Neovascularization of engineering bone in critical-sized condyle defects.

\begin{tabular}{lccccc}
\hline & \multicolumn{2}{c}{ 4 weeks } & & \multicolumn{2}{c}{12 weeks } \\
\cline { 2 - 3 } \cline { 5 - 6 } Group & Blood number/pore & Center region $(\%)$ & & Blood number/pore & Center region $(\%)$ \\
\hline hBMSCs & $87.48 \pm 12.87$ & $26.75 \pm 4.75$ & & $72.81 \pm 9.32$ & $25.82 \pm 3.64$ \\
hPCs & $78.33 \pm 10.45$ & $28.82 \pm 4.97$ & & $69.77 \pm 7.45$ & $23.76 \pm 5.79$ \\
hBMSCs+hPCs $(1: 1)$ & $82.59 \pm 7.85$ & $51.23 \pm 5.39^{\mathrm{a}, \mathrm{b}}$ & & $72.36 \pm 5.14$ & $37.32 \pm 4.85^{\mathrm{a}, \mathrm{b}}$ \\
hBMSCs+hPCs $(1: 2)$ & $86.75 \pm 9.77$ & $49.85 \pm 5.88^{\mathrm{a}, \mathrm{b}}$ & & $70.42 \pm 6.12$ & $33.29 \pm 5.37^{\mathrm{a}, \mathrm{b}}$ \\
hBMSCs+hPCs $(2: 1)$ & $79.56 \pm 11.46$ & $44.75 \pm 6.58^{\mathrm{a}, \mathrm{b}}$ & & $68.39 \pm 8.19$ & $32.84 \pm 6.29^{\mathrm{a}, \mathrm{b}}$ \\
Blank $\beta$-TCP scaffold & $37.57 \pm 7.35^{\mathrm{a}, \mathrm{b}}$ & $29.23 \pm 3.97$ & & $30.84 \pm 4.97^{\mathrm{a}, \mathrm{b}}$ & $24.72 \pm 4.86$ \\
\hline
\end{tabular}

${ }^{\mathrm{a}} \mathrm{P}<0.05$ vs hBMSCs; ${ }^{\mathrm{P}}<0.05$ vs hPCs. hBMSCs, human bone marrow stem cells; hPCs, human periosteal-derived stem cells; $\beta$-TCP, $\beta$-tricalcium phosphate.

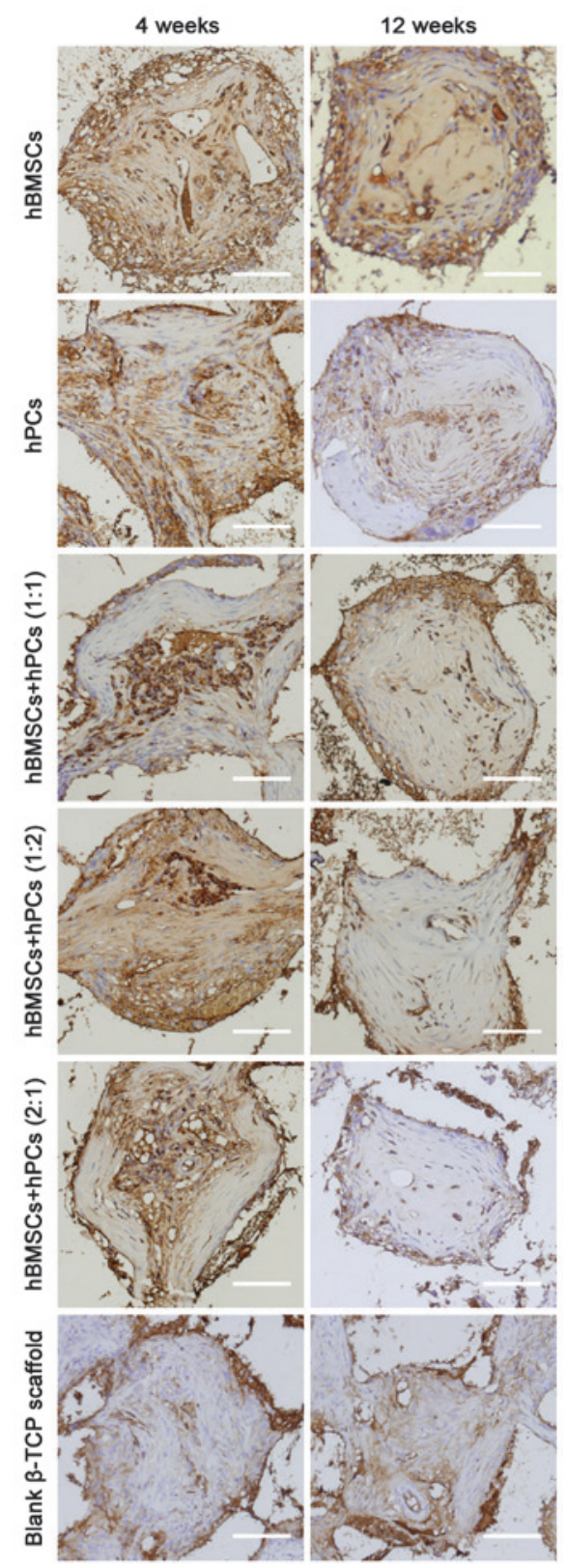

Figure 4. Neovascularization of femoral condyle implants was evaluated by vWF immunostaining (bar, $100 \mu \mathrm{m}$ ). hBMSCs, human bone marrow stem cells; hPCs, human periosteal-derived stem cells; $\beta$-TCP, $\beta$-tricalcium phosphate. scaffolds seeded with MSC vs. the controls (acellular $\beta$-TCP scaffolds). Co-culturing MSCs did not significantly enhance vascularization within the 3-D $\beta$-TCP scaffolds, compared with the hBMSCs and hPCs. Of note, however, all co-culturing MSCs demonstrated a remarkably higher percentage of blood vessels in the pore center region than that exhibited in the hBMSCs or hPCs (Table II).

\section{Discussion}

Engineering bone based on combined multipotent MSCs and a 3-D scaffold, presents a promising new strategy in bone regeneration that restores bony tissue following extensive loss as a result of trauma or disease (23-25). The identification of various types of MSCs from different ontological and anatomical sites has raised the question as to the optimal cellular source for such allogeneic applications. In the present study, hBMSCs and hPCs from the same donors were co-cultured. Their osteogenic potential was investigated using three different models (monolayer culture, 3-D culture and an in vivo model), in an attempt to determine whether this co-culturing strategy may be an alternative cellular source for hBMSCs and hPCs in cell-based approaches to bone repair. The results confirmed that, co-culturing hBMSCs with hPCs exhibits an overall enhanced capacity to differentiate towards the osteogenic lineage in vitro, which was reflected by robust mineralized node formation, steadily ALP positive staining and upgraded osteogenic-specific mRNA expression. Furthermore, for the repair of critical-sized femoral condylar defects, engineering bone constructed by co-culturing MSCs and porous $\beta-\mathrm{TCP}$ scaffolds exhibited noteably abundant, newly formed bone tissue, enhanced mature bone formation and increased center neovascularization, compared with the constructs seeded with hBMSCs or hPCs.

In the monolayer culture, in vitro osteogenic differentiation of co-culturing MSCs was determined by mineralized nodule formation and ALP staining. ALP is an early $\mathrm{m}$

arker of osteoblast differentiation, whereas mineralization of the matrix is associated with the late phase of osteoblast differentiation $(26,27)$. ALP is an ectoenzyme, produced by osteoblasts, that is involved in the degradation of inorganic pyrophosphate, providing sufficient local concentrations 
of phosphate or inorganic pyrophosphate for mineralization $(28,29)$. Therefore, ALP was used as a biochemical marker to determine osteoblast phenotype and it is considered as an important factor in determining bone differentiation (30-32). In addition, alizarin red $\mathrm{S}$ staining is the most common method of examining a mineralized matrix $(26,33)$. In the present study, during the early and late osteogenic differentiation stages, the number of ALP positive cells in the three co-culturing MSCs was higher than those in the hBMSCs or hPCs. Furthermore, it was confirmed that co-culturing hBMSCs and hPCs not only demonstrated abundant mineralized nodule formation, but also that alizarin red $\mathrm{S}$ positive staining appeared earlier than in the hBMCs and hPCs. The results suggest that co-culturing hBMSCs with hPCs strongly promotes MSC differentiation into their osteogenic phenotype and greatly enhances mineralized nodule formation in vitro.

For constructing engineered bone, biodegradable scaffolds are critical in providing 3-D space for the growth of osteogenic cells at an early stage and enough space for new bone formation when they are later degraded (34). Furthermore, the scaffolds offer a 3-D framework on which a temporary matrix, for cellular proliferation, differentiation and deposition of the ECM, facilitating the development of the neovasculature $(25,35)$. In the present study, the porous $\beta$-TCP scaffold was employed for the 3-D cultivation of MSCs. Its biocompatibility and its osteoconductive and osteoinductive features have been reported in several studies $(36,37)$. To further confirm the osteogenic capacity of co-culturing MSCs in 3-D cultivation, the osteogenic-specific mRNA expression of COL1 $\alpha 1$, BMP-2, OPN and OC were evaluated using qPCR. In skeletal tissues, the cells are distributed within a dense ECM composed of collagens, proteoglycans, a complex mixture of phosphoproteins and other inorganic materials (38). COL1 1 1 is an essential element in bone formation and it contributes to matrix production (39). During osteogenic differentiation, markers of the osteoblast phenotype appear, including the accumulation of extracellular bone matrix proteins, of which COL I is the most prevalent (32). OC is the most abundant non-collagenous protein in bone and is a useful tool as a bone-specific marker for terminal osteoblast differentiation. OC is released by calcified tissue and expressed at the late stage of differentiation $(40,41)$. OPN is a phosphorylated glycoprotein, recognized as a early marker of osteogenic differentiation and involved in the regulation of bone development and calcification (42). BMP-2 is secreted in an autocrine and paracrine fashion, and acts to directly induce differentiation of the MSCs into osteoblasts and to initiate the differentiation of osteoprogenitors from the host tissue into bone-forming cells (20). BMP-2 also serves as an osteoinductive signal, to increase infiltration and recruitment of surrounding repair cells, to further enhance bone regeneration and induce blood vessel ingrowth (43-45).

In 3-D cultivation, the results demonstrated that all three ratios of co-culturing MSCs significantly enhanced the mRNA expression of COL $1 \alpha 1$, OPN, OC and BMP-2 at different times. OPN and COL1 $\alpha 1$ were expressed at the early osteogenic inductive stage, however there was a discrepancy in that OPN mRNA transcripts were increased in a time-dependent manner, whereas COL $1 \alpha 1$ mRNA was steadily decreased following seven days in culture. The results suggest that the OPN mRNA was expressed earlier than the independent calcification. This may indicate that COL1 $\alpha 1$ participates in the initiation of osteogenic differentiation but does not directly participate in late-stage osteoblastic mineralization. Although gene expression of OC and BMP-2 in this 3-D cultivation were detected in the early osteogenic differentiation stage, their noteable expressions were recorded following 14 days in culture and their levels increased as calcification progressed from days 14 to 21 . These results indicate that the expression of OC and BMP-2 are closely correlated to biomineralization or hydroxyapatite crystallization. Based on these findings in 3-D cultivation, it may be confirmed that co-culturing hBMSCs with hPCs not only enhances the synthesis of the ECM and accelerates osteoblastic mineralization, but also stimulates osteoinductive signals to increase osteogenic differentiation.

The results from in vitro osteogenic differentiation and the capacity of new bone regeneration in vivo followed the same trends. For repairing critical-sized femoral condyle defects, co-culturing hBMSCs and hPCs demonstrated not only synergetic effects in promoting new bone formation, but greatly enhanced mature bone formation in vivo. This phenomenon is consistent with in vitro studies where co-culturing MSCs facilitated osteoblastic mineralization and upgraded osteogenic-specific mRNA expression. Furthermore, co-culturing MSCs revealed the formation of large quantities of bone tissue in the central (scaffolded) portion of the pore, whereas in the hBMSCs and hPCs alone, the newly formed bone was confined mainly to the margin of the pore.

Bone tissue is a complex and highly vascularized tissue. Angiogenesis is an essential component of normal bone development and fracture healing. Therefore, the evaluation of angiogenic activity in bone tissue engineering is important. MSCs are reportedly able to contribute directly to the formation of new blood vessels (46). The high degree of neovascularization facilitated the delivery of oxygen and nutrients for the construct and eventually contributed to the volume of tissue-engineered bone. It is also considered that adequate oxygen tension and a supply of other nutrients result from neovascularization, which allows direct formation of the mineralized matrix inside the scaffold (47). In addition, signals of newly formed vessels may positively affect the osteogenic potential of MSCs, which eventually affects the maturation of tissue-engineered bone (48). The results of the present study indicate that $\beta$-TCP scaffolds loaded with MSCs increase blood vessel ingrowth more than acellular scaffolds at the femoral condyle defect site. Furthermore, it was confirmed that co-culturing hBMSCs and hPCs notably facilitates central vascularization in the scaffold pores. These results may explain why co-culturing MSCs demonstrated a significantly higher percentage of new bone formation in the central pore region of the scaffold.

In conclusion, the present study first confirmed that MSCs, isolated from bone marrow and periosteum, have synergetic effects on osteogenic differentiation at the level of monolayer and 3-D cultivation, as well as on the critical-sized femoral condyle defects model. Co-culturing hBMSCs and hPCs not only increased osteoblastic mineralization and upgraded osteogenic specific mRNA expression, but it accelerated bone regeneration and enhanced mature bone formation. In addition, co-culturing hBMSCs and hPCs greatly facilitated central vascularization in the scaffold pores. Based on these 
findings, we recommend co-culturing hBMSCs and hPCs as a promising cellular source for bone-tissue engineering applications. Further study is necessary to clarify the exact mechanism of this synergetic effect on osteogenesis and vascularization.

\section{Acknowledgements}

This study was supported by the Natural Science Foundation of China (no. 81271998, 81271961, 81071452). The authors are grateful to Bio-lu Biomaterials Corp for supplying the $\beta$-TCP ceramic blocks.

\section{References}

1. Goulet JA, Senunas LE, DeSilva GL and Greenfield ML: Autogenous iliac crest bone graft. Complications and functional assessment. Clin Orthop Relat Res: 76-81, 1997.

2. Ferrara JL and Yanik G: Acute graft versus host disease: pathophysiology, risk factors, and prevention strategies. Clin Adv Hematol Oncol 3: 415-419, 428, 2005.

3. Lietman SA, Tomford WW, Gebhardt MC, Springfield DS and Mankin HJ: Complications of irradiated allografts in orthopaedic tumor surgery. Clin Orthop Relat Res: 214-217, 2000.

4. Bruder SP, Jaiswal N, Ricalton NS, Mosca JD, Kraus KH and Kadiyala S: Mesenchymal stem cells in osteobiology and applied bone regeneration. Clin Orthop Relat Res (Suppl): S247S256, 1998.

5. Srouji S, Maurice S and Livne E: Microscopy analysis of bone marrow-derived osteoprogenitor cells cultured on hydrogel 3-D scaffold. Microsc Res Tech 66: 132-138, 2005.

6. Arnsdorf EJ, Jones LM, Carter DR and Jacobs CR: The periosteum as a cellular source for functional tissue engineering. Tissue Eng Part A 15: 2637-2642, 2009.

7. Barachini S, Trombi L, Danti S, et al: Morpho-functional characterization of human mesenchymal stem cells from umbilical cord blood for potential uses in regenerative medicine. Stem Cells Dev 18: 293-305, 2009.

8. Campagnoli C, Roberts I A, Kumar S, Bennett PR, Bellantuono I and Fisk NM: Identification of mesenchymal stem/progenitor cells in human first-trimester fetal blood, liver, and bone marrow. Blood 98: 2396-2402, 2001.

9. De Coppi P, Bartsch G Jr, Siddiqui MM, et al: Isolation of amniotic stem cell lines with potential for therapy. Nat Biotechnol 25: 100-106, 2007.

10. Derubeis AR and Cancedda R: Bone marrow stromal cells (BMSCs) in bone engineering: limitations and recent advances. Ann Biomed Eng 32: 160-165, 2004.

11. Fickert S, Fiedler J and Brenner R E: Identification, quantification and isolation of mesenchymal progenitor cells from osteoarthritic synovium by fluorescence automated cell sorting. Osteoarthritis Cartilage 11: 790-800, 2003.

12. Zuk PA, Zhu M, Mizuno H, et al: Multilineage cells from human adipose tissue: implications for cell-based therapies. Tissue Eng 7: 211-228, 2001.

13. Mueller SM and Glowacki J: Age-related decline in the osteogenic potential of human bone marrow cells cultured in three-dimensional collagen sponges. J Cell Biochem 82: 583-590, 2001.

14. Phinney DG, Kopen G, Righter W, Webster S, Tremain N and Prockop DJ: Donor variation in the growth properties and osteogenic potential of human marrow stromal cells. J Cell Biochem 75: 424-436, 1999.

15. Eyckmans J, Luyten FP: Species specificity of ectopic bone formation using periosteum-derived mesenchymal progenitor cells. Tissue Eng 12: 2203-2213, 2006.

16. McDuffee LA and Anderson GI: In vitro comparison of equine cancellous bone graft donor sites and tibial periosteum as sources of viable osteoprogenitors. Vet Surg 32: 455-463, 2003.

17. Colnot C: Skeletal cell fate decisions within periosteum and bone marrow during bone regeneration. J Bone Miner Res 24: 274-282, 2009.

18. Solchaga LA, Cassiède P and Caplan AI: Different response to osteo-inductive agents in bone marrow- and periosteum-derived cell preparations. Acta Orthop Scand 69: 426-432, 1998.
19. Guichet JM, Braillon P, Bodenreider $\mathrm{O}$ and Lascombes P: Periosteum and bone marrow in bone lengthening: a DEXA quantitative evaluation in rabbits. Acta Orthop Scand 69: 527-531, 1998.

20. Huang YC, Kaigler D, Rice KG, Krebsbach PH and Mooney DJ: Combined angiogenic and osteogenic factor delivery enhances bone marrow stromal cell-driven bone regeneration. J Bone Miner Res 20: 848-857, 2005.

21. Jaquiéry C, Schaeren S, Farhadi J, et al: In vitro osteogenic differentiation and in vivo bone-forming capacity of human isogenic jaw periosteal cells and bone marrow stromal cells. Ann Surg 242: 859-867, 2005.

22. Giavaresi G, Fini M, Salvage J, et al: Bone regeneration potential of a soybean-based filler: experimental study in a rabbit cancellous bone defects. J Mater Sci Mater Med 21: 615-626, 2010.

23. Mistry AS and Mikos AG: Tissue engineering strategies for bone regeneration. Adv Biochem Eng Biotechnol 94: 1-22, 2005.

24. Otto WR and Rao J: Tomorrow's skeleton staff: mesenchymal stem cells and the repair of bone and cartilage. Cell Prolif 37: 97-110, 2004.

25. Salgado AJ, Coutinho OP and Reis RL: Bone tissue engineering: state of the art and future trends. Macromol Biosci 4: $743-765,2004$.

26. Igarashi M, Kamiya N, Hasegawa M, Kasuya T, Takahashi T and Takag M: Inductive effects of dexamethasone on the gene expression of Cbfa1, Osterix and bone matrix proteins during differentiation of cultured primary rat osteoblasts. J Mol Histol 35: 3-10, 2004.

27. Park BW, Hah YS, Kim DR, Kim JR and Byun JH: Osteogenic phenotypes and mineralization of cultured human periosteal-derived cells. Arch Oral Biol 52: 983-989, 2007.

28. Wang J, Asou Y, Sekiya I, Sotome S, Orii H and Shinomiya K: Enhancement of tissue engineered bone formation by a low pressure system improving cell seeding and medium perfusion into a porous scaffold. Biomaterials 27: 2738-2746, 2006.

29. Weinreb M, Shinar D and Rodan GA: Different pattern of alkaline phosphatase, osteopontin, and osteocalcin expression in developing rat bone visualized by in situ hybridization. J Bone Miner Res 5: 831-842, 1990.

30. Marom R, Shur I, Solomon R and Benayahu D: Characterization of adhesion and differentiation markers of osteogenic marrow stromal cells. J Cell Physiol 202: 41-48, 2005.

31. Stucki U, Schmid J, Hämmerle CF and Lang NP: Temporal and local appearance of alkaline phosphatase activity in early stages of guided bone regeneration. A descriptive histochemical study in humans. Clin Oral Implants Res 12: 121-127, 2001.

32. Wang H, Li Y, Zuo Y, Li J, Ma S and Cheng L: Biocompatibility and osteogenesis of biomimetic nano-hydroxyapatite/ polyamide composite scaffolds for bone tissue engineering. Biomaterials 28: 3338-3348, 2007.

33. Park BW, Hah YS, Kim DR, Kim JR and Byun JH: Vascular endothelial growth factor expression in cultured periostealderived cells. Oral Surg Oral Med Oral Pathol Oral Radiol Endod 105: 554-560, 2008.

34. Yuan J, Cui L, Zhang WJ, Liu W and Cao Y: Repair of canine mandibular bone defects with bone marrow stromal cells and porous beta-tricalcium phosphate. Biomaterials 28: 1005-1013, 2007.

35. Rai B, Oest ME, Dupont KM, Ho KH, Teoh SH and Guldberg RE: Combination of platelet-rich plasma with polycaprolactone-tricalcium phosphate scaffolds for segmental bone defect repair. J Biomed Mater Res A 81: 888-899, 2007.

36. Marino G, Rosso F, Cafiero G, Tortora C, Moraci M, Barbarisi $\mathrm{M}$ and Barbarisi A: Beta-tricalcium phosphate 3-D scaffold promote alone osteogenic differentiation of human adipose stem cells: in vitro study. J Mater Sci Mater Med 21: 353-363, 2010.

37. Neamat A, Gawish A and Gamal-Eldeen AM: beta-Tricalcium phosphate promotes cell proliferation, osteogenesis and bone regeneration in intrabony defects in dogs. Arch Oral Biol 54: 1083-1090, 2009.

38. Zheng YX, Ringe J, Liang Z, Loch A, Chen L and Sittinger M: Osteogenic potential of human periosteum-derived progenitor cells in PLGA scaffold using allogeneic serum. J Zhejiang Univ Sci B 7: 817-824, 2006.

39. Ignatius A, Blessing H, Liedert A, et al: Tissue engineering of bone: effects of mechanical strain on osteoblastic cells in type I collagen matrices. Biomaterials 26: 311-318, 2005. 
40. Bilkay U, Tokat C, Helvaci E, Ozek C, Zekioglu O, Onat T and Songur E: Osteogenic capacities of tibial and cranial periosteum: a biochemical and histologic study. J Craniofac Surg 19: 453-458, 2008.

41. Stein GS, Lian JB, Gerstenfeld LG, Shalhoub V, Aronow M, Owen T and Markose E: The onset and progression of osteoblast differentiation is functionally related to cellular proliferation. Connect Tissue Res 20: 3-13, 1989.

42. Giachelli CM and Steitz S: Osteopontin: a versatile regulator of inflammation and biomineralization. Matrix Biol 19: 615-622, 2000.

43. Bouletreau P J, Warren SM, Spector JA, Peled ZM, Gerrets RP, Greenwald JA and Longaker MT: Hypoxia and VEGF up-regulate BMP-2 mRNA and protein expression in microvascular endothelial cells: implications for fracture healing. Plast Reconstr Surg 109: 2384-2397, 2002.
44. Liang G, Yang Y, Oh S, et al: Ectopic osteoinduction and early degradation of recombinant human bone morphogenetic protein-2-loaded porous beta-tricalcium phosphate in mice. Biomaterials 26: 4265-4271, 2005.

45. Wozney JM: The bone morphogenetic protein family and osteogenesis. Mol Reprod Dev 32: 160-167, 1992.

46. Dufourcq P, Descamps B, Tojais NF, et al: Secreted frizzled-related protein-1 enhances mesenchymal stem cell function in angiogenesis and contributes to neovessel maturation. Stem Cells 26: 2991-3001, 2008.

47. Karageorgiou V and Kaplan D: Porosity of 3-D biomaterial scaffolds and osteogenesis. Biomaterials 26: 5474-5491, 2005.

48. Zhou J, Lin H, Fang T, Li X, Dai W, Uemura T and Dong J: The repair of large segmental bone defects in the rabbit with vascularized tissue engineered bone. Biomaterials 31: 1171-1179, 2010 . 